PAPER

\section{Serum lipids and memory in a population based cohort of middle age women}

\author{
V W Henderson, J R Guthrie, L Dennerstein
}

J Neurol Neurosurg Psychiatry 2003;74:1530-1535

See end of article for
authors' affiliations
$\ldots \ldots \ldots \ldots \ldots \ldots \ldots \ldots . \ldots . \ldots . \ldots$
Correspondence to:
VW Henderson, Donald
W Reynolds Center on
Aging, University of
Arkansas for Medical
Sciences, 4301 W.
Markham Street, \# 810,
Little Rock, Arkansas
72205 USA;
vhenderson@uams.edu
Received
19 November 2002
Revised accepted
12 April 2003 (....................
S erum lipids may affect cognition, but data are sparse and controversial. In relatively young adults, higher concentrations of total cholesterol (TC) and low density lipoprotein cholesterol (LDL-C) are associated with better performance on some cognitive tasks. ${ }^{12}$ However, lipid elevations also promote atherosclerosis, ${ }^{3}$ a suspected risk factor for cognitive decline in older persons, ${ }^{4}$ and may predispose to dementia..$^{5-7}$

Interventions designed to reduce lipid elevations are often implemented during midlife in otherwise healthy adults. To determine whether serum lipid levels are related to memory in this age group, we analysed longitudinal and cross sectional data from a population based cohort of middle age women. Based on previous studies in healthy, relatively young adults, ${ }^{128}$ we hypothesised that higher TC and LDL-C concentrations, but not high density lipoprotein cholesterol (HDL-C) or triglyceride concentrations, would be associated with better memory. Low TC has been linked to suicide risk and depression, ${ }^{9-12}$ and in secondary analyses we examined the relation between serum lipids and mood.

\section{METHODS}

\section{Subjects}

The Melbourne Women's Midlife Health Project (MWMHP) cohort was established in 1991 when a sample of Australian born women residing in the Melbourne metropolitan area and aged 45-55 years were recruited by random digit telephone dialling. ${ }^{13}$ Following baseline telephone interviews, 779 women were eligible for longitudinal studies based on having menstruated in the previous three months, having a uterus and at least one ovary, and not taking hormone therapy for menopausal symptoms or contraception: 438 $(56 \%)$ agreed to participate. Compared with women who declined to participate, these women were better educated, more likely to be employed, more likely to report good health, more likely to exercise regularly, and more likely to have had a Papanicolaou smear. ${ }^{14}$ Studies were approved by the
Human Research Ethics Committee of the University of Melbourne, and participants provided written informed consent.

\section{Memory and mood assessment}

Memory and mood were assessed during the year eight annual visit. Of 387 women in the MWMHP cohort at that time, 326 participated in memory testing; 42 were ineligible because of surgical menopause, 16 had moved from the metropolitan area, 2 were too busy, and 1 was ill. Memory was assessed using a 10 item supraspan word list recall task. One or two syllable nouns were read aloud to participants for three immediate recall trials. ${ }^{15}$ An unannounced delayed recall trial took place five minutes later, after an intervening task. In the absence of an a priori prediction that lipid concentrations would affect immediate and delayed recall differently, analyses were based on a total memory score representing the sum of the four recall trials. Positive and negative mood were assessed as previously described. ${ }^{16}{ }^{17}$ Participants were presented with 10 positive and 10 negative adjectives and asked to rate whether they felt that way during the last week on a four point scale ranging from 0 (hardly ever) to 3 (most of the time). ${ }^{18}$

\section{Lipid concentrations}

During each annual assessment, fasting morning blood samples were taken for lipid measurements. ${ }^{19}$ TC, HDL-C, and triglycerides were measured using standard enzymatic methods on routine automated chemistry systems. Lipid analyses were validated against serum reference materials supplied by the Center for Disease Control Lipid

\footnotetext{
Abbreviations: TC, total cholesterol; LDL-C, low density lipoprotein cholesterol; HDL-C, high density lipoprotein cholesterol; MWMHP, Melbourne Women's Midlife Health Project
} 


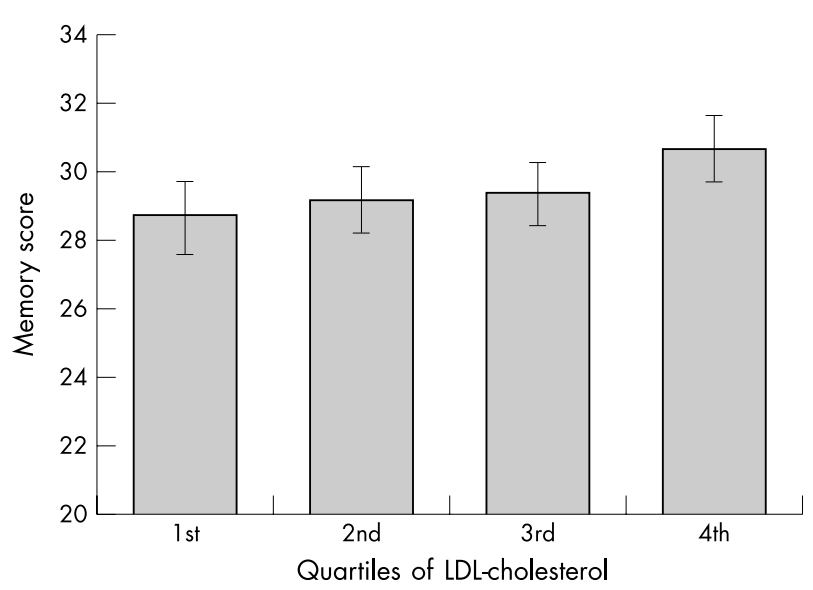

Figure 1 Mean memory scores according to quartiles of low density lipoprotein cholesterol concentration. Error bars represent 95\% confidence intervals. Pairwise comparisons confirmed better memory for women in the fourth low density lipoprotein cholesterol quartile compared with the first $(p=0.005)$ and second $(p=0.04)$ quartiles.

Standardization Program. LDL-C was calculated using the Friedewald formula, observing the restriction that this calculation is valid only up to triglyceride levels of $5 \mathrm{mmol} / \mathrm{l}^{20}$

\section{Control variables}

Self reported information collected from structured interviews or questionnaires during year 8 or previous assessments included age (calculated from the birth date), education, employment status, marital status, parity, menopausal status, medication use (including oestrogen containing hormone therapy and lipid lowering agents), smoking history, alcohol use, and exercise. Based on standard definitions, menopausal status was determined from reported changes in menstruation and prospectively maintained menstrual calendars. ${ }^{21}$ For hormone therapy, women were classified as never users, past users, or current users based on self reported use during annual assessments of oestrogen begun during or after the menopausal transition. Both adiposity ${ }^{22}$ and oestrogens ${ }^{23}$ influence serum lipid concentrations, and body mass index (body weight in kilograms divided by height in metres squared) and serum oestradiol concentration (measured as previously described ${ }^{14}$ ) were determined during the year 8 assessment.

\section{Statistical analyses}

Single explanatory variable analyses were undertaken before multivariable models. For memory and for positive and negative mood, values were modelled as a function of explanatory variables in multiple regression analyses. Analysis of covariance was used for categorical variables. Explanatory variables included current lipid levels, previous lipid levels, and changes in lipid levels compared with former years. We also explored possible effects of lipid lowering agents on memory. When memory was the dependent variable, control variables described above plus mood were considered as potential confounders. For positive or negative mood, control variables plus the memory score were potential confounders. To obtain more stable estimates of serum lipid concentrations, lipid levels were analysed as two year averages: years $7+8$ (representing current levels), years $4+5$, and years $1+2$. When values in years $7+8$ were significantly associated with memory performance, we then evaluated lipid values before years $7+8$. When three year changes between years $4+5$ and years $7+8$ were significantly associated with memory, we then evaluated six year lipid changes between years $1+2$ and years $7+8$. All probabilities were two tailed. Because of skewed distributions, triglyceride values were analysed after logarithmic transformation, and negative mood was analysed after square root transformation. Analyses were performed using SPSS for Windows, version 11.0.

\section{RESULTS}

Cohort characteristics for women with year 8 memory measures are shown in table 1 , and serum lipid levels are given in table 2 . Summed over four recall trials, the mean memory score was 29.5, standard deviation (SD) 4.4 (median 30). There were no ceiling or floor effects. One woman achieved a maximum score of 40; no participant scored 0 on any recall trial. The mean positive mood score was 22.7 (SD, 6.1 ; median, 24), and the mean negative mood score was 3.6 (SD, 3.3; median, 3).

\section{Lipids and memory}

In the year 8 assessment, memory was significantly, negatively associated with negative mood $(r=-0.18)$ and positively associated with education $(r=0.28)$, parity $(r=0.12)$ and alcohol use $(r=0.14)$. There were no associations with positive mood, age, paid employment, marital status, menopausal status (menopausal transition $v$ postmenopausal among women not using hormone therapy), use of hormone therapy (ever $v$ never, or current $v$ past $v$ never), use of lipid lowering drugs, smoking, exercise, body mass index, or serum oestradiol concentration (all p values $>0.1$ ). Except where noted, subsequent analyses investigating the relation between lipids and memory adjusted for potentially confounding effects of education, parity, alcohol use, and negative mood. The absence of significant association with age presumably reflected the restricted age range of MWMHP participants; however, age was included as an additional control variable because of its known association with memory in other studies.

\section{Current lipid concentrations and memory}

In multiple regression analyses, year $7+8$ LDL-C concentrations were significantly predictive of the memory score $(\beta=0.714, p=0.01)$. The association with TC was of near significance $(\beta=0.454, p=0.08)$, but there were no significant associations with levels of HDL-C $(\beta=0.036, p=1.0)$ or triglycerides $(\beta=-0.377, p=0.4)$. In exploratory analyses using analysis of covariance, LDL-C concentration quartiles differed significantly with respect to memory scores $F=2.92$, $\mathrm{p}=0.04$ ) (fig 1). In pairwise comparisons, the mean difference between first and fourth LDL-C quartiles on the memory task represented a difference of 0.44 standard deviations $(p=0.005)$.

\section{Previous LDL-C concentrations and memory}

To help determine whether observed cross sectional associations between memory and current LDL-C represented relatively short term effects of cholesterol concentrations or relatively long term effects, we examined the relation between memory and previous LDL-C concentrations. However, LDL-C from three years earlier did not predict memory scores $(\beta=0.245, p=0.4)$.

\section{Changes in lipid concentrations and memory}

To assess whether longitudinal changes in lipid levels affected memory, we analysed the difference between current levels and those from previous years. In multiple regression analyses that included baseline TC levels as a control variable, an increase in TC between years $4+5$ and years $7+8$, but not between years $1+2$ and years $7+8$, was positively related to memory $(p=0.01)$ (table 3$)$. In similar analyses, the change in serum LDL-C between years $4+5$ and years $7+8$ was 
Table 1 Clinical features of Melbourne's women midlife health project participants during the year 8 assessment*

\begin{tabular}{lll}
\hline & $\mathrm{N}$ & $\%$ \\
\hline Mean age (years) & 56.7 (SD 2.5) & Range: 52-63 \\
Body mass index $\left(\mathrm{kg} / \mathrm{m}^{2}\right.$ ) & 27.0 (SD 5.4) & Range: 16.2-57.1 \\
Education $>12$ years & 108 & $33 \%$ \\
Education $\leq 12$ years & 218 & $67 \%$ \\
Full or part time paid employment & 197 & $60 \%$ \\
Not employed & 129 & $40 \%$ \\
Married or living with partner & 252 & $77 \%$ \\
Not currently married or with a partner & 74 & $23 \%$ \\
O parity & 26 & $8 \%$ \\
1 or 2 parity & 131 & $40 \%$ \\
3 or more parity & 169 & $52 \%$ \\
Menopausal transition, no hormone therapy & 50 & $15 \%$ \\
Postmenopausal, no hormone therapy & 200 & $61 \%$ \\
Current hormone therapyt & 76 & $23 \%$ \\
Use of lipid lowering drugs & 29 & $9 \%$ \\
Current smoking & 47 & $14 \%$ \\
Never or past smoking & 279 & $86 \%$ \\
No alcohol during past week & 116 & $36 \%$ \\
1 or more alcoholic drinks during past week & 210 & $64 \%$ \\
Regular exercise once a week or less & 106 & $33 \%$ \\
Regular exercise 2 or 3 times a week & 91 & $28 \%$ \\
Regular exercise 4 or more times a week & 129 & $40 \%$ \\
\hline SD, standard deviation. & & \\
*326 women had memory assessed in year 8 and 306 had lipid determinations. & & \\
†64 other women were past users of hormone therapy. &
\end{tabular}

significantly predictive of the memory score $(p=0.003)$, whereas LDL-C differences between years $1+2$ and years $7+8$ were not (table 3 ). Compared with women whose LDL-C concentrations were unchanged or had increased between years $4+5$ and years $7+8$, the mean memory score was 0.39 standard deviations lower among LDL-C decliners $(\mathrm{p}=0.002)$ (table 3 ). For TC concentrations, the 0.24 standard deviation difference in memory scores between decliners and nondecliners was also significant $(p=0.04)$ (table 3$)$. In other analyses, there was no relation between memory and threeyear changes in HDL-C or triglyceride concentrations ( $p$ values $>0.1$ ).

When memory was analysed in relation to three year changes in TC grouped by quartiles, there were significant differences in analysis of covariance that adjusted for baseline TC levels and other control variables $(F=4.50$, $\mathrm{p}=0.004)$. Adjusted mean memory scores for the first to the fourth quartiles were 28.1, 29.4, 29.0, and 30.7, respectively. Pairwise comparisons showed that women in the fourth quartile, the group with largest increases in TC, had significantly better mean memory scores than women in the first $(p<0.0005)$ and third $(p=0.02)$ quartiles. For women in the fourth and first quartiles, there was a 0.59 standard deviation, difference in mean memory score.

Table 2 Serum lipid concentrations in cohort members*

\begin{tabular}{lllll}
\hline & $\begin{array}{l}\text { Total } \\
\text { cholesterol } \\
\text { (SD) }\end{array}$ & LDL-C (SD) & HDL-C (SD) & $\begin{array}{l}\text { Triglycerides } \\
\text { (SD) }\end{array}$ \\
\hline Years 1+2 & $5.83(0.90)$ & $3.77(0.89)$ & $1.58(0.41)$ & $1.07(0.72)$ \\
Years 4+5 & $5.83(0.89)$ & $3.64(0.88)$ & $1.62(0.40)$ & $1.28(0.92)$ \\
Years 7+8 & $5.84(0.91)$ & $3.79(0.87)$ & $1.49(0.39)$ & $1.30(0.79)$ \\
\hline
\end{tabular}

SD, standard deviation; LDL-C, low density lipoprotein cholesterol; HDL$C$, high density lipoprotein cholesterol.

*Values in $\mathrm{mmol} / \mathrm{I}$ were calculated from means of two annual samples. For total cholesterol, LDL-C, and $\mathrm{HDL}-\mathrm{C}$, to convert $\mathrm{mmol} / \mathrm{l}$ to $\mathrm{mg} / \mathrm{dl}$, multiply by 38.6 . For triglycerides, to convert $\mathrm{mmol} / \mathrm{l}$ to $\mathrm{mg} / \mathrm{dll}$, multiply by 88.5 . Years $1+2$ samples were obtained in both years from 313 women, in years $4+5$ from 309 women, and in years $7+8$ from 300 women.
When memory was analysed in relation to quartiles of LDL-C change between years $4+5$ and $7+8$, there were also significant differences among quartiles in analysis of covariance that adjusted for baseline LDL-C levels and other variables $(F=3.24, \mathrm{p}=0.02)$ (fig 2$)$. In post hoc comparisons, the mean difference of 2.1 points on the memory task between women in the fourth and first quartiles corresponded to a difference of 0.48 standard deviations $(\mathrm{p}=0.006)$.

Immediate recall versus delayed recall

A word list memory task involves several cognitive domains, and immediate recall may involve different abilities from recall after a short delay. Post hoc analyses considered the relation between TC and LDL-C levels and memory performance summed over the three immediate recall trials and separately for the fourth (delayed recall) trial. In multiple

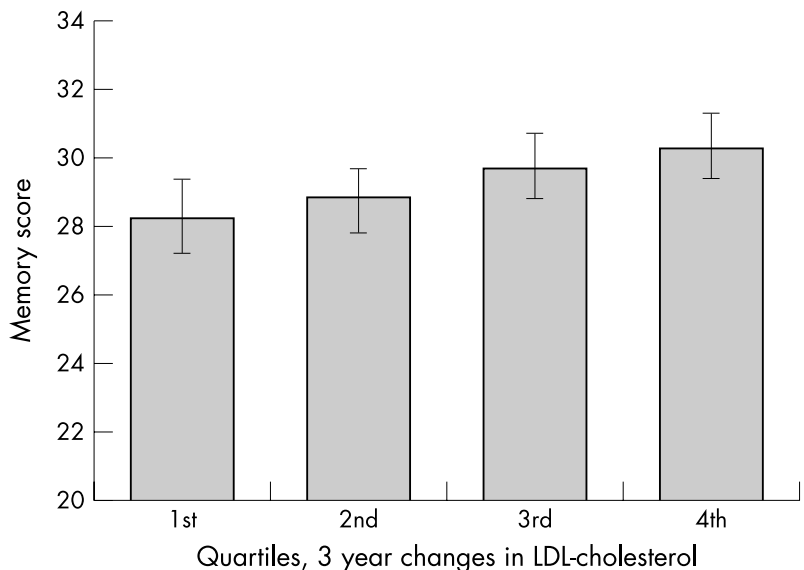

Figure 2 Mean memory scores according to 3 changes (Years 7+8 minus Years $4+5$ ) in low density lipoprotein cholesterol concentration quartiles. Error bars represent $95 \%$ CLs. Pairwise comparisons confirmed significantly better memory scores for women in the fourth low density lipoprotein cholesterol quartile compared with the first $(p=0.006)$ and second $(p=0.02)$ quartiles, and for women in the third quartile compared with the first $(p=0.05)$. 
Table 3 Memory and changes in total cholesterol or low density lipoprotein cholesterol concentrations*

\begin{tabular}{|c|c|c|c|c|c|c|}
\hline & \multirow[b]{2}{*}{$\beta$} & \multirow[b]{2}{*}{ Probability } & \multicolumn{4}{|c|}{ Memory score $\dagger$} \\
\hline & & & Decliners & $\begin{array}{l}\text { Non- } \\
\text { decliners }\end{array}$ & $\begin{array}{l}\text { Difference } \\
\text { Points }\end{array}$ & $\begin{array}{l}\text { Difference } \\
\text { z score }\end{array}$ \\
\hline \multicolumn{7}{|c|}{ Total cholesterol } \\
\hline & 0.891 & 0.01 & & 29.8 & $1.0 \S$ & 0.24 \\
\hline $\begin{array}{l}\text { change } \\
\text { six year } \\
\text { change }\end{array}$ & 0.370 & 0.3 & $\begin{array}{l}(n=131) \\
-\end{array}$ & $(n=161)$ & - & - \\
\hline \multicolumn{7}{|c|}{ Low density lipoprotein chole } \\
\hline $\begin{array}{l}\text { three } \\
\text { year }\end{array}$ & 1.224 & 0.003 & 28.3 & 30.0 & $1.7 \ddagger$ & 0.39 \\
\hline $\begin{array}{l}\text { change } \\
\text { six year }\end{array}$ & & 0.07 & $\begin{array}{l}(n=97) \\
-\end{array}$ & $\begin{array}{l}(n=187) \\
-\end{array}$ & & - \\
\hline
\end{tabular}

${ }^{*} \beta$ and probability values are based on multiple regression analyses with the memory score as the dependent variable and the change in total cholesterol level or low density lipoprotein cholesterol as the independent variable. Analyses were adjusted for control variables, as described in the text. The three year change was calculated as the difference between years $7+8$ and years $4+5$ lipid values; six year change was calculated as the difference between years $7+8$ and years $1+2$ values.

tMemory scores represent adjusted mean values. Decliners refers to women whose total cholesterol or low density lipoprotein cholesterol levels declined, and non-decliners refers to women whose levels did not decline. Differences between decliners and non-decliners were not evaluated for six year changes in total cholesterol and low density lipoprotein cholesterol, because six year changes were not significantly associated with memory.

$\S$ In analysis of covariance, $F=4.14, p=0.04$.

fln analysis of covariance, $F=9.22, p=0.002$.

$\mathrm{n}=$ number of women in each group.

regression analyses, years $7+8$ TC $(\beta=0.391, p=0.04)$ and LDL-C $(\beta=0.572, p=0.005)$ were significant predictors of immediate recall, but neither predicted delayed recall ( $\beta=0.067, p=0.5$ for TC; $\beta=0.145, p=0.1$ for LDL-C). Changes in TC and LDL-C levels between years $4+5$ and $7+8$ also predicted immediate but not delayed recall scores (for TC change, immediate recall $\beta=0.655, p=0.007$, and delayed recall $\beta=0.094, p=0.4$; for LDL-C change, immediate recall $\beta=0.742, p=0.008$, and delayed recall $\beta=0.150$, $\mathrm{p}=0.3)$.

Analyses excluding participants with poor memory MWMHP participants were not known to have dementia, but poor performance on delayed recall tasks is common among those likely to be diagnosed later with Alzheimer's disease. ${ }^{24}$ To help ensure that results were not inadvertently biased by the inclusion of dementia cases, analyses were repeated after post hoc exclusion of all women falling two or more standard deviations below the mean delayed recall score and, separately, after exclusion of those two or more standard deviations below the mean total memory score. The prediction of current TC concentrations for the total memory score remained of near significance after excluding women with poor delayed recall scores or excluding women with poor total memory scores (both $p$ values $\leqslant 0.06$ ), and current LDL$\mathrm{C}$ concentrations remained significantly predictive of memory (both $\mathrm{p}$ values $\leqslant 0.02$ ). The three year change in TC remained positively associated with memory (both $p$ values $=0.02$ ), and increases in LDL-C still significantly predicted memory when women with poor delayed recall scores or poor total memory scores were excluded ( both $\mathrm{p}$ values $\leqslant 0.006$ ).

\section{Lipids and mood}

Both positive $(r=0.22)$ and negative $(r=-0.15)$ mood were significantly associated with exercise; as noted above, negative mood was also associated with memory performance.
There were no significant or near significant associations with other control variables. In separate multiple regression analyses adjusting for effects of exercise and memory scores, current TC, LDL-C, HDL-C, or triglyceride levels were not predictive of positive mood or negative mood (all $p$ values $>0.1$ ). Similarly, there were no significant associations between three year lipid changes and mood (all p values $>0.1$ ). Post hoc analyses that excluded the memory score as a control variable did not alter results.

\section{Statin use, memory, and mood: post hoc analyses}

Use of prescription lipid lowering agents was reported by 29 women during year 8; all agents were $3 \beta$-hydroxy-3 $\beta$ methylglutaryl-coenzyme A reductase inhibitors (statins). Analyses of covariance compared statin users with other women. Statin use was not associated with memory, positive mood, or negative mood (all p values $>0.1$ ). Results did not change after further adjustment for TC levels.

\section{DISCUSSION}

Cholesterol, an integral component of cell membranes, modulates membrane protein function. ${ }^{25}$ The brain has a high lipid content, and dietary cholesterol directly influences brain cholesterol concentrations. ${ }^{26}$ For women in the MWMHP cohort, greater serum concentrations of LDL-C were positively associated with better memory performance. There was a non significant trend between current TC levels and the total memory score, but there was no association for HDL-C or triglyceride levels.

Research on the relation between serum cholesterol and cognition is inconsistent. Hypercholesterolaemia promotes atherosclerosis, ${ }^{3}$ and TC elevations are linked to vascular dementia. ${ }^{6}$ Higher TC and LDL-C levels were associated with lower scores on a global cognitive measure in an observational study of older women (mean age 71 years) with coronary heart disease. ${ }^{27}$ In a retirement community cohort, TC and LDL-C concentrations were higher among women (mean age 80 years) who performed poorly on a clock drawing task administered four years later. ${ }^{28}$ Although the association between TC and Alzheimer's disease is inconsistent, ${ }^{5-7}$ experimental cholesterol reduction lowers production of $\beta$-amyloid, ${ }^{29}$ a key biochemical abnormality in this disorder. ${ }^{30}$

Statin use is linked to reduced risk of dementia. ${ }^{31-33}$ It is not certain, however, that lowered cholesterol is causative, as the association is stronger for less potent statins ${ }^{31}$ and may not occur in other classes of lipid lowering agents, ${ }^{32}$ and because confounding by indication or cessation bias was not always confidently excluded in reported studies. ${ }^{34}$ In the MWMHP cohort, we observed no association between statin use and memory independent of lipid concentrations; speculatively, anti-inflammatory and antioxidant actions of statins are more relevant to dementia than lipid lowering effects. ${ }^{35}$

The relation between cholesterol and memory in the elderly may differ from that in younger or middle age adults, where prevalences of atherosclerosis and dementia are lower. Present results thus support previous reports that higher concentrations of TC or LDL-C are associated with better cognition, particularly for tasks dependent on mental manipulation or rapid mental processing. ${ }^{128}$ In university students, choice reaction times were worse in women with lower TC. ${ }^{1}$ In healthy volunteers (mean age 44 years), higher TC and LDL-C concentrations were associated with better performance on a timed visuoconstructive task (block design test) but not on tasks that depend primarily on previously acquired knowledge (information and vocabulary). ${ }^{2}$ In a small five year longitudinal study of monozygotic twin pairs (mean age 55 years) discordant for decline on a digit symbol 
substitution task, TC levels were significantly lower among decliners. $^{8}$

In the MWMHP cohort, the magnitude of memory score variance attributed to LDL-C concentration was small (2\%) but not negligible. Education accounted for over $6 \%$ of the variance and negative mood for $2.5 \%$; other identified control variables were less important than LDL-C. On the memory task, women in the lowest LDL-C quartile scored more than two fifths of a standard deviation below those in the highest quartile (fig 1). By way of comparison, in the Heart and Estrogen/progestin Replacement Study of older women with known cardiovascular disease, women in the lowest LDL-C quartile compared with those in the highest quartile scored about one fourth standard deviation higher on a global cognitive measure. ${ }^{27}$

The association between LDL-C and memory was stronger for serum concentrations measured close to the time of memory testing than for levels obtained three years before. Putative effects of LDL-C on memory therefore reflect more recent levels. However, three year changes in TC and LDL-C concentrations were also significantly related to memory (table 3), accounting for about $2 \%$ (TC) or $3 \%$ (LDL-C) of memory score variance. Memory scores of women whose LDL-C levels declined during this three year interval were 0.39 SD lower than scores of women whose levels had remained the same or increased (table 3). Results were similar in post hoc analyses comparing LDL-C changes between women in the first and fourth quartiles (fig 2).

Not all studies agree, ${ }^{36}$ but low TC is associated with suicide risk and depression. ${ }^{9-12}$ We were unable to detect a link between serum lipids and mood in women without diagnosed depression. Similarly, interventions that reduce cholesterol do not affect mood. ${ }^{37} 38$

In post hoc analyses, effects of TC and LDL-C concentrations on memory-as well as effects of three year changes in concentrations of these lipids-were more pronounced for immediate recall than delayed recall. Both tasks involve long term episodic memory. For immediate recall from a supraspan word list, cognitive demands might also include attentional and organisational skills involved in working memory and executive functions. It is unknown why TC and LDL-C effects were evident only for immediate recall, but this finding supports previous claims that cholesterol elevation is linked to better performance on tasks where information processing demands are greater. ${ }^{2}$

Clinical trial data also provide partially consistent findings. In a 12 week randomised trial involving hypercholesterolaemic adults (mean age 53 years), participants receiving dietary interventions performed significantly worse than controls on a sustained attention task (but not on several other tasks) ${ }^{37}$; decrements in sustained attention correlated with declines in serum TC. ${ }^{37}$ In another randomised trial, hypercholesterolaemic patients (mean age 46 years) treated with statins for six months showed small but significant performance decrements on tests of attention and psychomotor speed; memory was unaffected in this study. ${ }^{38}$ In older women with heart disease, statin use is linked to better cognition, but interestingly this association is unrelated to lipid levels. ${ }^{27}$

LDL-C is routinely calculated from $\mathrm{TC},{ }^{20}$ and TC and LDL-C are therefore not independent determinations. Our findings from LDL-C were generally more robust than those from TC, and LDL-C is probably the critical lipid variable influencing memory scores. Whether this association is causal, however, cannot be determined from our data.

It is unlikely that our findings were influenced by inclusion of women with early dementia. MWMHP participants were not old enough to face a substantial risk of dementia (table 1), and the significant association between LDL-C and memory was unaltered in post hoc analyses excluding women who scored poorly on delayed recall or on the total memory score. Although pathological changes of Alzheimer's disease may begin years before the onset of frank dementia, ${ }^{39}$ our findings indicate that higher LDL-C levels in women may protect memory at midlife.

Our results are derived from a well characterised population based cohort where blood samples and a portion of the clinical data were collected prospectively. Limitations of our findings include their observational nature, the absence of additional cognitive measures, and the absence of male participants. The $\epsilon 4$ allele of apolipoprotein $\mathrm{E}$ influences serum lipid concentrations ${ }^{40}$ and Alzheimer's disease risk, ${ }^{30}$ but apolipoprotein E genotyping was not performed. Public health implications of our results are uncertain, given the well documented vascular risks in later life associated with TC and LDL-C elevations. ${ }^{3}$ However, medications that reduce serum cholesterol are widely prescribed, ${ }^{41}$ and long term consequences of TC and LDL-C reductions on cognitive functioning are unknown. Future trials that evaluate cholesterol lowering interventions in healthy adults should consider possible effects on cognition.

\section{ACKNOWLEDGMENTS}

We thank Dr John Taffe for advice on statistical analyses, Ms Emma Dudley for database management and preliminary statistical analyses, and Mr Nicholas Balazs for comments on lipid assessment methodologies.

Research was supported in part by grants from the Public Health \& Research Development Committee of the National Health \& Medical Research Council of Australia, the Victorian Health Promotion Foundation, and the Alzheimer's Association (IIRG-01-2684).

\section{Authors' affiliations}

V W Henderson, Departments of Geriatrics, Neurology, Pharmacology \& Toxicology, and Epidemiology, University of Arkansas for Medical Sciences, Little Rock, Arkansas, USA

J R Guthrie, L Dennerstein, Office for Gender and Health, Department of Psychiatry, University of Melbourne, Parkville, Australia

Portions of this research were conducted while the first author was Visiting Professor, Department of Psychiatry, University of Melbourne, and the Kearney Visiting Professor at the Mental Health Research Institute of Victoria

Competing interest: LD has received a grant in aid from Pfizer Pharmaceuticals. WWH and JRG have no competing interests to declare

\section{REFERENCES}

1 Benton D. Do low cholesterol levels slow mental processing? Psychosom Med 1995;57:50-3

2 Muldoon MF, Ryan CM, Matthews KA, et al. Serum cholesterol and intellectual performance. Psychosom Med 1997;59:382-7.

3 Ross R. Mechanisms of disease: atherosclerosis - an inflammatory disease. N Engl J Med 1999;340:115-26.

4 Haan MN, Shemanski L, Jagust WJ, et al. The role of APOE epsilon4 in modulating effects of other risk factors for cognitive decline in elderly persons. JAMA 1999;282:40-6.

5 Notkola IL, Sulkava R, Pekkanen J, et al. Serum total cholesterol, apolipoprotein E $\epsilon 4$ allele, and Alzheimer's disease. Neuroepidemiology 1998;17:14-20.

6 Moroney JT, Tang MX, Berglund L, et al. Low-density lipoprotein cholesterol and the risk of dementia with stroke. JAMA 1999;282:254-60.

7 Evans RM, Emsley CL, Gao S, et al. Serum cholesterol, APOE genotype, and the risk of Alzheimer's disease: a population-based study of African Americans. Neurology 2000;54:240-2.

8 Swan GE, LaRue A, Carmelli D, et al. Decline in cognitive performance in aging twins: heritability and biobehavioral predictors from the National Heart, Lung, and Blood Institute twin study. Arch Neurol 1992;49:476-81.

9 Lindberg G, Rastam L, Gullberg B, et al. Low serum cholesterol concentration and short term mortality from injuries in men and women. BMJ 1992:305:277-9.

10 Modai I, Valevski A, Dror S, et al. Serum cholesterol levels and suicidal tendencies in psychiatric inpatients. J Clin Psychiatry 1994;55:252-4.

11 Kunugi H, Takei N, Aoki H, et al. Low serum cholesterol in suicide attempters. Biol Psychiatry 1997;41:196-200.

12 Morgan RE, Palinkas LA, Barrett-Connor E, et al. Plasma cholesterol and depressive symptoms in older men. Lancet 1993;341:75-9. 
13 Dennerstein L, Smith AMA, Morse CA, et al. Menopausal symptomatology: the experience of Australian women. Med J Aust 1993;159:232-6.

14 Burger HG, Dudley EC, Hopper JL, et al. The endocrinology of the menopausal transition: a cross-sectional study of a population-based sample. $J$ Clin Endocrinol Metab 1995;80:3537-45.

15 Collie A, Shafiq-Antonacci R, Maruff P, et al. Norms and the effects of demographic variables on a neuropsychological battery for use in healthy ageing Australian populations. Aust N Z J Psychiatry 1999:33:568-75.

16 Dennerstein $\mathrm{L}$, Lehert $\mathrm{P}$, Burger $\mathrm{H}$, et al. Mood and the menopausal transition. J Nerv Ment Dis 1999; 187:685-91.

17 Dennerstein L, Lehert P, Dudley E, et al. Factors contributing to positive mood during the menopause transition. J Nerv Ment Dis 2001;189:84-9.

18 Kammann R, Flett R. Affectometer 2: a scale to measure current level of general happiness. Australian Psychology 1983;35:259-65

19 Shelley JM, Green A, Smith AMA, et al. Relationship of endogenous sex hormones to lipids and blood pressure in mid-aged women. Ann Epidemio 1998;8:39-45

20 Friedewald WT, Levy RI, Fredrickson DS. Estimation of the concentration of low-density lipoprotein cholesterol in plasma, without use of the preparative ultracentrifuge. Clin Chem 1972;18:499-502.

21 Taffe J, Dennerstein L. Retrospective self-report compared with menstrual diary data prospectively kept during the menopausal transition. Climacteric 2000;3:183-91.

22 Vermeulen A, Verdonck L. Sex hormone concentrations in post-menopausal women: relation to obesity, fat mass, age and years post-menopause. Clin Endocrinol (Oxf) 1978;9:59-66.

23 Lobo RA. Effects of hormonal replacement on lipids and lipoproteins in postmenopausal women. J Clin Endocrinol Metab 1991;52:156-8.

24 Petersen RC, Smith GE, Waring SC, et al. Mild cognitive impairment: clinical characterization and outcome. Arch Neurol 1999;56:303-8.

25 Yeagle PL. Modulation of membrane function by cholesterol. Biochimie 1991;73:1303-10.

26 Howland DS, Trusko SP, Savage MJ, et al. Modulation of secreted $\beta$-amyloid precursor protein and amyloid $\beta$-peptide in brain by cholesterol. J Biol Chem 1998;273:16576-82.

27 Yaffe K, Barrett-Connor E, Lin F, et al. Serum lipoprotein levels, statin use, and cognitive function in older women. Arch Neurol 2002;59:378-84.
28 Paganini-Hill A, Henderson VW. The effects of hormone replace therapy, lipoprotein cholesterol levels, and other factors on a clock drawing task in older women. J Am Geriatr Soc 1996;44:818-22.

29 Refolo LM, Pappolla MA, LaFrancois J, et al. A cholesterol-lowering drug reduces beta-amyloid pathology in a transgenic mouse model of Alzheimer's disease. Neurobiol Dis 2001;8:890-9.

30 Selkoe DJ. Alzheimer's disease: genes, proteins, and therapy. Physiol Rev 2001:81:741-6.

31 Wolozin B, Kellman W, Ruosseau P, et al. Decreased prevalence of Alzheimer disease associated with 3-hydroxy-3-methyglutaryl coenzyme $A$ reductase inhibitors. Arch Neurol 2000;57:1439-43.

32 Jick H, Zornberg GL, Jick SS, et al. Statins and the risk of dementia. Lancet 2000;356:1627-31.

33 Rockwood K, Kirkland S, Hogan DB, et al. Use of lipid-lowering agents, indication bias, and the risk of dementia in community-dwelling elderly people. Arch Neurol 2002:59:223-7.

34 Haley RW, Dietschy JM. Is there a connection between the concentration of cholesterol circulating in plasma and the rate of neuritic plaque formation in Alzheimer disease? Arch Neurol 2000;57:1410-12.

35 Vaughan CJ, Delanty N. Neuroprotective properties of statins in cerebral ischemia and stroke. Stroke 1999:30:1969-73.

36 Brown SL, Salive ME, Harris TB, et al. Low cholesterol concentrations and severe depressive symptoms in elderly people. BMJ 1994;308:1328-32.

37 Wardle J, Rogers $\mathrm{P}$, Judd $\mathrm{P}$, et al. Randomized trial of the effects of cholesterol-lowering dietary treatment on psychological function. Am J Med 2000; 108:547-53.

38 Muldoon MF, Barger SD, Ryan CM, et al. Effects of lovastatin on cognitive function and psychological well-being. Am J Med 2000;108:538-47.

39 Elias MF, Berger A, Wolf PA, et al. The preclinical phase of Alzheimer's disease: a 22-year prospective study of the Framingham cohort. Arch Neurol 2000:57:808-13.

40 Eggersten G, Tegelman R, Ericsson S, et al. Apolipoprotein E polymorphism in a healthy Swedish population: variation of allele frequency with age and relation to serum lipid concentrations. Clin Chem 1993;39:2125-9.

41 Top 200 brand-name drugs by units in 2001. DrugTopics. Com: The Online News Magazine for Pharmacists, March 4, 2002. www.drugtopics.com. Accessed January 2003

\section{$\mathrm{ECHO}$}

\section{The apolipoprotein E phenotype is not a visual loss susceptibility gene in Leber hereditary optic neuropathy $\beta$}

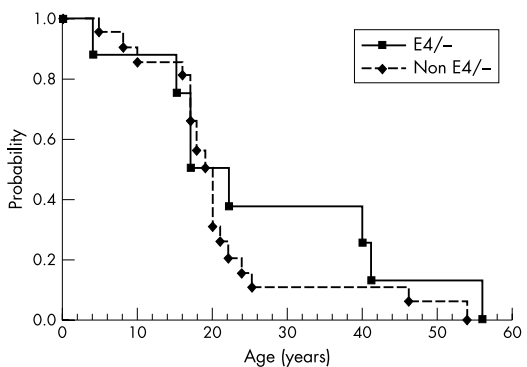

Figure 1 Effect of $A P O E \in 4$ allele on age of onset of visual failure in LHON pedigrees from the north east of England ( $Y$ axis indicates probability of remaining unaffected).
$T$ he apolipoprotein E phenotype $(A P O E)$ is known to be strongly associated with several neurodegenerative conditions including Alzheimer disease and dementia with Lewy bodies. Since $A P O E$ is produced by Muller cells within the retina and rapidly transported to the optic nerve, researchers from Newcastle upon Tyne (UK) and Milan (Italy) postulated that the genotype might modulate the expression of the primary mtDNA mutations in Leber Hereditary Optic Neuropathy (LHON).

Sixteen pedigrees from the UK and 44 from Italy were ascertained and investigated with family controls who were unaffected carriers. The authors found no effect of the specific mtDNA mutation on the risk of visual failure and no evidence of a significant effect of the genotype upon disease penetrance. The age of onset of blindness was no different in those with or without the $\epsilon 4 /-$ and non- $\epsilon 4 /$ - genotypes.

It remains possible the genotype may have a subtle effect, for example by affecting the chance of visual recovery after the acute phase.

A Journal of Medical Genetics 2003;40:e41

(http://www.jmedgenet.com/cgi/content/full/40/4/e41)

$\Delta$ Please visit the Journal of Neurology, Neurosurgery, and Psychiatry website [www.jnnp.com] for a link to the full text of this article. 